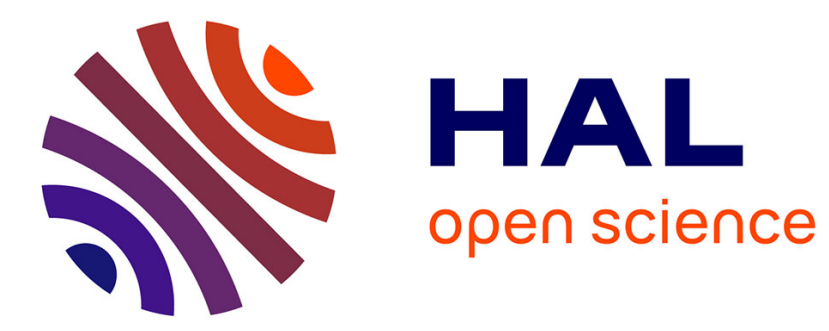

\title{
Cortical basis of communication: local computation, coordination, attention
}

Frédéric Alexandre

\section{To cite this version:}

Frédéric Alexandre. Cortical basis of communication: local computation, coordination, attention. Neural Networks, 2009, 22, pp.126-133. inria-00335971

\section{HAL Id: inria-00335971 \\ https://hal.inria.fr/inria-00335971}

Submitted on 31 Oct 2008

HAL is a multi-disciplinary open access archive for the deposit and dissemination of scientific research documents, whether they are published or not. The documents may come from teaching and research institutions in France or abroad, or from public or private research centers.
L'archive ouverte pluridisciplinaire HAL, est destinée au dépôt et à la diffusion de documents scientifiques de niveau recherche, publiés ou non, émanant des établissements d'enseignement et de recherche français ou étrangers, des laboratoires publics ou privés. 


\title{
Cortical basis of communication: local computation, coordination, attention
}

\author{
Frederic Alexandre \\ LORIA-INRIA BP 239 \\ F-54506 Vandoeuvre, France \\ frederic.alexandre@loria.fr, \\ http://www. loria.fr/ ${ }^{\sim}$ falex
}

\begin{abstract}
Human communication emerges from cortical processing, known to be implemented on a regular repetitive neuronal substratum. The supposed genericity of cortical processing has elicited a series of modeling works in computational neuroscience that underline the information flows driven by the cortical circuitry. In the minimalist framework underlying the current theories for the embodiment of cognition, such a generic cortical processing is exploited for the coordination of poles of representation, as it is reported in this paper for the case of visual attention. Interestingly, this case emphasize how abstract internal referents are built to conform to memory requirements. This paper proposes that these referents are the basis for communication in humans, which is firstly a coordination and an attentional procedure with regard to their congeners.
\end{abstract}

Key words: Computational neurosciences, Communication, Visual attention, Coordination, Model of the cortex

\section{Introduction}

The cerebral cortex is known to be composed of millions of similar elementary circuits, repeated throughout its surface, the cortical columns [8, 23, 4]. A fascinating challenge with the study and modeling of the cortex is to understand how the wide spectrum of functions generated by this ultimate structure in the evolution process can emerge from this repetitive paving. The corresponding approach of modeling in Computational Neuroscience is 
to consider the cortical column as the adequate level of computation [2] and to define its functioning and learning rules in such a way that whatever the cortical area or processing pathway we consider, only the nature of the inputs and the size and connectivity scheme of the considered cortical regions will specify its resulting activity.

Three kinds of data from Neuroscience are requested to guide modelers in this domain. At the microscopic level, it is fundamental to be informed about the inner structure of the cortical column, seen as a circuit of a hundred of neurons, including its inputs, outputs and dynamics. At the mesoscopic level, the organization of a network of automata, proposed as models of cortical columns, must be consistent with current knowledge about the main information flows in the brain. Columns must be gathered in cortical areas; cortical areas must be interconnected with a precise connectivity scheme; the cortical processing is also frequently dependent on extra-cortical information flows (including perceptive input and motor output) that have to be defined at this level. At the macroscopic level, behavioral studies are also necessary to figure out what kind of information is processed and how it is manipulated, stored and exploited to interact with the outer world.

Other non bio-inspired theories in machine intelligence are perhaps more intuitionist. They rely on a logic, sequential and structural approach and have a tendency to build symbolic and abstract representations of information (models of the world), whereas the distributed, local, numerical and asynchronous approach in Computational Neuroscience is rather slanted toward the emergence of properties and toward the intensive use of interactions with the real world to build the minimal necessary internal representations. Experiments from psychophysics and other developmental issues are therefore necessary not to follow our misleading intuition.

In this paper, my goal is to explain that current studies modeling cortical processing with distributed simple local computations can be directly used to emulate complex behaviors related to communication. I will report several studies that have been carried out to define the local functioning of a cortical automaton (section 2). Elementary behavioral functions using such local computations will then be presented in section 3. I will refer to them as coordination functions since they allow for the linkage of representational poles in the cortex. Section 4 will report recent developments of a more ambitious study about mechanisms of selective attention. One of the goals of that study is to show that elaborated functions like attention, central in the cognition and often presented as related to consciousness [31], can emerge 
from the distributed asynchronous computations of local automata and can avoid many representational hypotheses difficult to implement and justify. Similarly, the claim of this paper is that communication capabilities can also emerge from the same distributed asynchronous computations. In section 5, I will argue that an artificial system, endowed with a distributed substratum of computation inspired with the local mechanisms of cortical circuitry presented here, could be able to exhibit some fundamental communication capabilities as observed in evolved living being. Section 6 will conclude on the amount of work that remains to be done to obtain more elaborated communication channels like observed in spoken language.

\section{Principles of local distributed computation}

The local circuitry in the cortex has been studied for a long time. Many studies have laid emphasis on the cortical column, seen as the elementary computing block of the cortex $[8,23,4,16,20]$. The cortical column is perpendicular to the surface of the cortex and goes through the six layers of the cortex. A very basic and functional description of these layers can be proposed as follows: Layer 4 receives extra-cortical (thalamic) and feedforward cortical input; layers 1, 2 and 3 are cortico-cortical connections; and layers 5 and 6 send feedback in the cortex and projections outside the cortex. Apart from the granular layer 4, all these projections are performed by pyramidal excitatory neurons and other excitatory and inhibitory interneurons achieve the internal logic of the automaton, coupling and uncoupling these layers. The interplay between these information flows is the basis of neural field approaches developed by many authors [36, 32, 3, 29]. Others have proposed a cortical automaton including several inputs and outputs and internal learning and functioning rules $[16,2,20]$.

From an evolutionary perspective, the cortex can be seen as a new evolved loop, added to more primitive perception-action loops [4]. In this view, the cortex offers a substratum characterized by modal maps where specific information can be displayed, by associative areas where subtle intermodal dependencies can be expressed and by an original relaxation dynamics [9]. These powerful properties are obtained from the layered organization of the cortex. They can be described through four principles of information processing, as described in the next subsections and summarized in Table 1. 


\subsection{Thalamic input and feedforward}

Thalamic and cortical feedforward integration is certainly the most classical principle of cortical functioning. Cortical columns in sensory areas are known to be selective to specific stimuli and to perform filtering on the incoming perceptive information, from thalamus to primary sensory areas or from primary to secondary sensory areas. Kohonen has popularized this operation with the self-organizing map principle [19]. It combines adaptive feedforward links conveying thalamic inputs and learning relevant filters with static lateral inhibition implementing a competition between filters. More precise and developmental details have been introduced by Miikkulainen [22] and Taylor has proposed a dynamic and distributed version of such a processing, where bubbles of activity are generated and maintained from an initial stimulation [32].

The learning rule proposed by Kohonen highlights the main principles of plasticity related to the thalamic feedforward information flow. The cortex plays a role of reduction of dimensionality from the huge set of possible thalamic inputs to a reduced set of prototypes selected to represent the statistical distribution of the inputs. Prototypical learning (quantization) selects the best representatives. Lateral inhibition ensures their sparseness. The bidimensional nature of the cortex allows for the emergence of a topography where close units are selective to close stimuli [19].

\subsection{Lateral propagation of information within an area}

Apart from stereotyped bell-shaped (eg with DOG: difference of Gaussians) pattern of connection allowing for competition within population of close columns (and evoked in the previous subsection), lateral connectivity within cortical areas plays an important role in temporal information processing in the cortex $[17,6]$. Events and stimuli in the world have an important temporal dimension and representing only static stimuli is not sufficient. Spatio-temporal self-organization mechanisms have been proposed, which exploit lateral excitatory connectivity in cortical areas [35]. The principle can be illustrated with the image of drops of water falling on a liquid surface: consider a sequence of events, each event stimulating a cortical unit. For event $\mathrm{n}$, the stimulation of a cortical unit will generate a lateral wave of excitation within the map. This wave will collide with the waves emitted by the cortical units excited by events $n-1$ and $n+1$. Cortical units situated at the collision

loci of the waves will be able to learn the corresponding co-excitation and 
consequently the sequence of activation within the map. A bayesian mechanism has been proposed [18] for the learning of such sequences. It also enables anticipation, with the pre-activation of the next event in the sequence. Its principle relies on a Hebbian learning between the thalamic or feedforward activation of the unit representative of an event and the cortical activation of the unit representative of the preceding event in the sequence.

This mechanism of lateral propagation of excitation is described here as encoding sequences of events but it could have a more general usefulness. This processing principle of the cortex is not yet fully mastered but seems to be a fundamental property to be better understood. For example, the cerebellum shares some structural properties with the cortex (tilled with similar micro-circuits) but does not have such a lateral connectivity [9]. Its appearance in the cortex could have endowed it with dynamic relaxation properties, allowing for propagation of waves on its surface for the search of solutions to multiple constraints expression [8].

\subsection{Cortico-cortical connections}

Connections between cortical areas have been studied for two general cases of activity: multi-modal integration and feedback projections. In both cases, the problem is to interconnect two cortical maps representing information of different kinds, either to create a mixed representation of both (multi-modality) or to enable one map to be controled or monitored by the activity of the other (feedback).

A large amount of work has studied multi-modality as well as feedback, most of which is somewhat consistent as far as functioning and learning rules

are concerned. The general idea is to advocate for the implementation of a gating effect between maps. Differently from afferent connections having an integrative effect onto cortical units, such cortico-cortical connections will rather have a multiplicative influence onto existing activity in the map: a strong signal will enhance the activity whereas a weak signal will inhibit it. Such a logic has been frequently implemented in sigma-pi units [1, 12] for which Hebbian learning rules can be easily derived.

\subsection{Sub-cortical output and feedback}

Competition and association between cortical columns yield a pattern of activation in cortical maps that has to be transformed into actions or decisions in the outer world or into a feedback influence onto lower maps. This is the role of deeper layers 5 and 6 of the cortex, particularly in the 
motor and premotor cortex. Layers 5 and 6 also establish feedback links between hierarchical sensory areas.

\subsection{In summary}

We have presented here some principles of information processing generally considered as responsible for cortical organization and involving the laminar architecture of the cortex. This functional view defines a basic automaton of computation, the cortical column, considered as the crossroad of several information flows in the cortex. Local specialization and coupling and uncoupling in the automaton enhance functional circuits. We now illustrate how elementary behavioral functions exploit these mechanisms.

\section{Coordination}

Many models of the cerebral cortex have been illustrated by applications devoted to the discrimination of perceptive stimuli (e.g. visual recognition) and to the orientation of actuators in space (e.g. target reaching). These two kinds of tasks illustrate a fundamental property of the cortex, structured into two pathways [15]. The parietal axis (where pathway) which associates perceptive and body poles of the cortex. And the temporal axis (what pathway) which associates the perceptive and the limbic poles of the cortex, the latter being known to be the locus of multi-modal episodic memory [30] (pathway toward the hippocampus).

\subsection{Identification and location}

Identification and location might appear as rather different tasks. Particularly, imagining that they emerge from the same local logic of computation and that only the nature of the data and the structure of the network specify the task can appear as a rather difficult exercise. Effectively, even if many models are consistent with the general principles mentioned above, their detailed implementation generally makes identification and location models incompatible. This is not acceptable in the framework of the unified theory of cortical processing that this paper tries to promote. I first explain here why the tasks are not so different as they could appear at first sight and then propose a framework of interpretation that could correct some excesses which in my opinion are often made in classical models.

As mentioned by Norman [24] and by Goodale and Humphrey [15], it is important to underline that identification and location tasks have been 
designed by researchers from different origin and with different goals. Identification is a classical task in statistics and artificial intelligence (e.g. classification, recognition tasks) and the general goal of such a task is to activate only one output unit (the class number) for each possible perceptive input. Location rather refers to geometrical and behavioral aspects and the goal is here to compute, within a population of neurons, transformations from one reference frame to another. Moreover in this latter case, the underlying task is more ecological and compatible with Gibsonian theories [14], known to be difficultly compatible with symbolic artificial intelligence promoted by the former domain.

Are these theories and principled approaches only a distorting mirror which hides the similarity of the tasks? The first argument for a positive answer comes from a description of the tasks in terms of information flows. First of all, it must be reminded that parietal and temporal axis can be seen as providing an association between different poles of representation in the cortex. In a more dynamical view, it can be said that they coordinate poles of representation. Basically, the parietal axis performs a coordination between perceptive (visual, auditory) and body representation, whereas the temporal axis performs a coordination between perceptive stimuli and the inner representations that they trigger in the limbic system (attractive vs repellent; love vs hate; etc.). In both cases, learning the coordination corresponds to extract hints that are discriminant in the association between the poles into consideration. Later, this learning can be used to activate a population of neurons to orient the body toward a target or to activate few neurons representative of a class to be recognized, but in both cases, the same kind of distributed network establishing relations between poles of representation is exploited (cf Table 2). This unified view of coordination between poles of representation promotes the principle of purposive perception [15]: the axes of processing depend on the purpose of the poles to be associated and consequently determine the hints to be extracted in the intermediate representations.

Ballard and colleagues [5] have illustrated such a dual view in a task of identification or location of stimuli. From high-dimensional feature vectors extracted from the image of objects, they first explain how a neuronal distribution of activity can be memorized in internal representations. Then, they underline the similarity of identification and location tasks: Identification corresponds to build a neuronal activation from the current foveal view, to compare it with a large number of memorized internal representations and 
to select the one best matching the current view. Location corresponds to activate only one internal representation (the object we wish to locate in the scene), to find the place in the visual field with the closest neuronal activation and to return its location. In both cases, the resources are the same but they are transformed by the way they are used.

\subsection{The situatedness of cognition}

The most synthetic way to reconcile identification and location is to remember that the phenomenon we are interested in here, the human cognition, emerges from the interactions of the body with the world. As evoked above, the body can orient toward objects to better perceive and analyze them. The analysis is performed on the basis of physical measurements and more or less elaborated emotional reactions of the body generated by the stimuli. This will elicit other orientation activities of the body (approach and grasping or avoidance). This elementary behavior is made possible by the capacity to coordinate the perceptive representation of the outer world (including the perception of the body itself) with the answer that it generates either in the physical body reference frame (parietal axis) or in the more emotional reference frame linked to the limbic system (temporal axis). In both cases, this capacity of coordination is acquired from experience and interaction with the world. In this perspective, the cortex is seen as a multi-modal device, learning the self-organization of the various frames of representation sent to it by the outer (perception, proprioception) and the inner (emotion, rhythms) world, and also the self-organization of the associative substratum linking the poles of representation. Menard and his colleagues have shown that a self-organization process can also act on such multi-modal information. This process displays information in such a way that it builds in associative areas an intermediate representation between the poles [21].

In the elementary tasks mentioned here (identification and location), only two pathways are considered but the same explanation apply to other more complicated behaviors. Particularly, the frontal lobe of the cortex is known to stand for the motor pole of representation [13]. Thanks to its links with the basal ganglia and the posterior part of the cortex, the prefrontal cortex associates the consequences of motor action with sensory information from the inner and outer world, self-organized in the posterior part of the cortex as mentioned above, and combines this information with goal oriented rewards computed in the basal ganglia to decide the temporal organization of behavior which best satisfies the constraints. This aspect is out of the scope of this 
paper and will be only evoked in the concluding part. Nevertheless, it is worth mentioning that the present approach also claims that units in the prefrontal cortex obey the same rules of local computation and global organization in poles of representation, as in the posterior cortex.

Moreover, the theory of the situatedness of cognition gives other principles about information representation which are of high interest to understand which relations and coordinations are learned in the cortex. Opposite to intellectualist symbolic approaches and their abstract, complex and hierarchical representations, the situatedness of cognition proposes to minimize representational contents [25] and privileges simple strategies, more directly coupling perception and action [7] and more efficient to react quickly in the changing environment.

A key aspect of this theory of intelligence is the Gibsonian notion of affordance [14]: perception is not a passive process and, depending on the current task, objects are discriminated as possible tools that could be used to act in the environment. Whereas a scene full of details can be memorized in very different and costly ways, a task-dependent description is a very economical way that implies minimal storage requirements. Hence, remembering becomes a constructive process.

Ballard and his colleagues [5] have done a decisive step to operationalize this concept. From the idea that the orientation of the body is important for cognition, they propose that eye movements, performed at an average rate of one third of a second, are the right level of description of the computational interface between neuronal and behavioral processes: the embodiment level. They propose to consider eye fixation as a deictic pointer (with the same meaning and usefulness as in computer science) that binds variables extracted from the environment. In this framework, the meaning of the pointer corresponds to task-relevant components of the object being fixated. It must be underlined how, as in computer science, the pointer mechanism is clever and economical: the pointer can be manipulated internally for complex operations. For the same operation to be performed on different objects, it is not necessary to store all the details in memory but just to change the referent of the pointer. Also, with such a deictic strategy, the organism can keep track of relevant targets in the environment by only storing the movement of the eye necessary to foveate it. We do not memorize details of the object but we know which eye movement to perform to get them: The world itself is considered as an external memory.

All these principles can possibly be applied to coordination tasks (identi- 
fication and location) but are not necessary for modelers working at the level of only one processing pathway: it is still possible for them to be influenced by a more symbolic approach and to build a fully detailed representation of information; the system is generally effective and only its biological plausibility can be discussed. The same cannot be said about more complex tasks, where several processing pathways and poles of representation are implicated. My opinion is that such systems are difficult to build and master with a too rich representation of information just because this approach is not sustainable, as it is demonstrated at this level of complexity. In the next section, I present some experiments, based on the embodiment of cognition, which successfully implement complex tasks with a minimal representation.

\section{Attention}

We now consider more complex tasks, involving several frames of representation and, in the framework of the above theory, several pointers to index the world. In this section, among the variety of body orienting movements, real and virtual eye movements only are considered: fixation and attention, seen as mechanical and neural deictic devices.

Concerning fixation, Ballard and colleagues [5] have deeply studied an experimental procedure, in which a subject has at his disposal a set of colored blocks to copy a model onto a workspace. Behavioral studies reveal the strategy generally chosen by subjects, faithful to the minimal internal memory principle and described by a set of eye movements. One typical iteration of the block copying task can be described as follows: the eye goes to the model to acquire the color of the next block to be copied (identification), goes to the set of blocks to find one with the same color (location), the hand picks up the foveated block while the eye goes back to the model to acquire the position of the considered block; the hand with the block moves toward the workspace and drops the block at the place where, meanwhile, the eye has indicated the similar place as in the model. It can be remarked that this multiple fixation strategy draws minimally on internal memory by binding objects to variables, at the cost of a longer execution time. This behavioral study is directly linked to several computational models, related to computer vision and reinforcement learning but, as far as I know, not to connectionist approaches. I now present a connectionist model that we are currently building, based on such a unified theory of cortical processing. This model aims 
at implementing covert and overt visual attention, as a way to internally and externally manage eye movements and their related deictic properties.

\subsection{Local functioning rules}

The model is composed of a set of two dimensional discrete neural fields, which are direct extensions of the model proposed by Amari [3], in 2D and with a discrete implementation of topological maps. Each of the maps corresponds to a cortical area, composed of units corresponding to cortical automata, as presented above. As described below and sketched in Figure 1, a set of maps is implemented to represent two information flows, the Where and the What pathways, receiving external information from a visual input (retina) and able to internally focus on a target and to produce camera movements to center a desired target in the middle of the retina (fovea).

As a consequence, at the level of the cortical automaton, several kinds of input and output have to be specified. The feedforward flow between areas and the lateral flow within areas are integrated with weighted summations. Cortico-cortical connections between areas are implemented by a multiplicative rule (weighted sum of the product of activity of afferent neurons). The weighting functions are chosen as a Gaussian for the lateral flows and as a difference of Gaussians for the feedforward flows. They are constant for the cortico-cortical connections. At the moment, these weights are fixed from biological data [22]; current work aims at defining learning rules to adapt them.

\subsection{Emerging properties}

From these local uniform functioning rules, several interesting properties emerge from a structured network, depending only on the connectivity.

Saliency: The feedforward weights can be chosen in such a way that, in a topological map receiving an external visual input, each unit responds best to a stimulus in a given receptive field and with a given orientation or color. This allows to implement Feature maps (cf. Figure 1) acting as topological filters on these features. These elementary feature maps converge onto the Saliency map which displays a multimodal representation. In all these maps, the Gaussians are narrow and several stimuli can be represented. Gaussians are larger in the Focus map that receives information from the Saliency map. As a consequence, a stronger competition takes place in the Focus map and only one blob of activity can survive. This competition can be biased by feedback information from one map in the What axis (Feature processing 
axis in Figure 1) which can specify one feature of the desired target (eg: a red pattern or orientation on the left). Conversely, the Focus map can bias the What axis in order to display the identity of the pattern that win the competition in the Focus map (eg: red pattern oriented on the left).

Inhibition of return: If the goal of the task is to successively focus onto each pattern with specified features, a mechanism is desired to prevent the system from going several times to the same place. This mechanism is known as the inhibition of return [26] and is implemented with a couple of maps called Working memory in Figure 1 and described in [34]. One map in the Working memory receives topological (one to one) excitations from the Focus and the Saliency maps and sends reciprocal excitatory topological connections to another similar map that helps to maintain excitation: both external inputs are necessary to create excitation but only one (from the Saliency map) is sufficient to maintain it. This cumulative mechanism creates a dynamical memory of the locations that have been explored. When the focus of attention has to change ('switch' order in the covert mode), this memory will inhibit the Focus map and force it to look for another location.

Anticipation: A camera movement completly modifies the visual reference frame and all that have been built in the working memory could be lost. To avoid this perturbating phenomenon, a Memory anticipation map is built (cf. Figure 1). It is computed as a convolution product of the Working memory and the Focus map. Just before a movement is triggered, the Focus map gives the information of the next location that will be attended. Thanks to the multiplicative effect of the Memory anticipation map, the activity of the Working memory is translated by the movement vector represented in the Focus map. When the camera movement is performed, this prediction of the future state of the working memory meets the feedforward activity sent by the Saliency map and the representation remains valid and stable.

\subsection{Integrated behavior}

The properties evoked above are only a functional interpretation of the collective behavior of few interconnected maps of cortically-inpired automata. This level of interpretation can also be used to relate the whole system to such a complex behavior as visual attention.

Covert attention does not imply eye movements and allows for parallel feature search and sequential conjunction search (involving several features) with inhibition of return. Treisman and Gelade [33] explain this property in their Feature Integration Theory where a global feedforward processing 
is only possible inside one feature map and an iterative sequential process including feedback operates in the conjunctive cases.

An initial model [34] including the Focus and Working memory maps is able to perform covert attention and displays similar properties [10]: if the desired target is encoded only in a feature map, it can directly bias the competition in the Focus map; else a selection corresponding to only one feature dimension is made, must be compared to the desired target and eliminated (thanks to the inhibition of return) or selected (hence a search time proportional to the number of targets).

We have recently extended the system (adding the Memory anticipation map) to allow for eye movements (overt attention). This is consistent with the premotor theory of attention [27] that considers that covert and overt attentions share several neuronal structures. As eye movements perturbate the frames of representation, there is a need to anticipate the consequences of eye movements to update the working memory [11], as illustrated in Figure 2. Here the goal is to center the retina on a blue target. In the figure, the red square corresponds to the retina and contains a blue target at the bottom. This target is selected by the focus map; in the Anticipation map, it is anticipated that the target will be in the middle of the retina and the Working memory will be translated accordingly, as the movement is performed.

In this system, the visual search can be also much more complex; it is for example possible to sequentially focus on all patterns with the same characteristics, the search for one pattern being itself a conjunctive search. In this case, for example, the goal would be to focus on all blue bars with a $\mathrm{Pi} / 4$ orientation. First, the covert attention will successively make more salient several Blue or $\mathrm{Pi} / 4$ patterns until one with both characteristics in found (else, the Switch neuron eliminates a bad candidate with inhibition of return and ask for the next candidate). When a matching candidate is found, over attention can apply and ask for a real camera movement to translate this pattern in the middle of the retina. Just before the movement, the Memory anticipation mechanism will anticipate the modification in the frame of representation and modify the Working memory accordingly. Here too, it must be underlined that this functional interpretation emerges from distributed asynchronous computations of cortically inspired maps of units.

This mechanism can also be interpreted in the framework of the situatedness of cognition. In the "feature axis" corresponding to the temporal axis, a kind of saliency map uses a filtering process to choose targets from intrinsic characteristics of perceived objects or because they are "desired" 
(the goal of the task). Then, on this basis, in the "spatial axis" standing for the parietal axis, only the locations of stimuli, that can be considered as pointers toward objects in the real world, are manipulated. Particularly, a gating mechanism using sigma-pi units combines the activity of a focus map indicating the next target to focus on with the activity of the working memory to anticipate what should be the state of the working memory after the eye movement (cf. figure 1 and refer to [12] for details). After this internal process of pointer manipulation, when the eye movement is performed, it is possible to relate this information about location to the real objects in the world and to their characteristics.

Figure 2 illustrates the model running in a simplified environment, where the behavior of the system can be finely observed. Current work complexifies the input filters to apply the system to natural images. Concerning the size of the current model, it should be underlined that it consists of eighteen maps, fifteen thousands units and over six millions connections, which illustrates the fact that fully distributed asynchronous computations can be mastered until that level, provided that a solid theoretical framework ensures the consistency of the whole.

\section{Communication}

In the preceding sections, I have presented the principles of a theory of intelligence based on the situatedness of cognition, together with several behavioral and biological related facts. I have shown the interests of such a framework for tasks related to visual scene exploration with or without eye movements. The main goal of this paper is to argue that this framework is also fundamental to endow artificial beings with efficient and bio-inspired communication skills.

It was indicated above that the orientations of any parts of the body can be used as deictic pointers toward the real world and that this principle is used by the human being to keep track of relevant "objects" (with a broad meaning) in the world. Firstly, since all humans use that strategy, consciously or unconsciously observing the orientation of the body parts of other humans can be useful to be informed about their intentionality: This is a first way

to communicate. Secondly, the spoken language can be integrated to that framework if we go beyond the visual modality put to the fore for the moment. From the multi-modal integration principles evoked at the beginning of this paper, it can be understood that an "object" that is evoked, observed 
or manipulated can be associated with an articulated sound. Similarly to pointers to the outer world, this sound can also be considered as a pointer to the object it used to be associated with.

Concerning non-verbal communication, referents can be achieved by pointing with the hand, the head, the eyes, etc. and the listener is able to bind the real object to those deictic pointers (e.g.: do it where I am fixating). Concerning spoken communication, deictic pointers are useful to interpret indexicals (e.g.: this is mine). In both cases, we find again that principle to represent information from hints toward the outer world as it is needed rather than to build complete and complex internal representations of the world.

All this phenomenological description particularly matches with what we know about the role of mirror neurons in communication [28]. Besides, they are ideally located at the crossroads of multimodal sensory information and action representation in the cortex. They are also characterized by a generic spectrum of responses, independent from the agent and the sensory modality and, as part of the cortex, share the same structuration in cortical columns with the other parts of the cortex.

\section{Perspectives}

The preceding section is itself a prospective part because a lot of work remains to be done to operationalize such concepts. Nevertheless, it seems to me that the way forward is rather clear to proceed in the domain. The main prerequisite is to be able to operate several (artificial and/or real) agents creating, observing and manipulating such deictic pointers in a real world.

It must be also underlined that I mainly developed here the first level of the theory, based on deictic strategies at a third of a second or so, concerning orientations of parts of the body or uttered words. A lot remains to be done to consider behaviors with longer constants of time, requiring manipulations and combinations of elementary pointers. Similarly to computer science, it is possible to imagine creating pointers of pointers and preserving the genericity of the computations. Two cerebral mechanisms must be evoked in that perspective. Firstly, the perirhinal cortex and the hippocampic system are reputed to store high level abstract representations [30]. Secondly, the prefrontal-striatal-thalamic-cortical loops own a variety of time constants that allow for the organization of behavior in time respecting several kinds

of constraints [13]. At the moment, I have described separated elements of 
behavior. Such mechanisms are necessary to integrate them in behaviors with longer constant of time while preserving their reactivity and flexibility. First, this is necessary to develop longer term dynamical memory (to have the illusion of a stable world in the visual case; to remember the general meaning of the sentence while uttering a word). Second, this is necessary to give a stronger meaningful structure to the behavior (intelligent exploration of the world in the visual case; syntactic rules in language). My claim is that such powerful processes could emerge from these mechanisms and that these mechanisms could be implemented with the same local computational logic as the one described in this paper.

Finally, concerning both aspects, they should be developed in parallel to solid knowledge concerning the underlying cerebral circuitry. Here also, there exists a number of evidence, evoked above, relating most aspects to posterior and prefrontal cortex, hippocampus and basal ganglia. Modeling experiments could also bring new predictions to be confronted to the biological and behavioral reality. 


\section{References}

[1] F. Alexandre and F. Guyot. Neurobiological Inspiration for the Architecture and Functioning of Cooperating Neural Networks. In Proceedings International Workshop on Artificial Neural Networks, Malaga (Spain), June 1995.

[2] F. Alexandre, F. Guyot, J.-P. Haton, and Y. Burnod. The Cortical Column: A New Processing Unit for Multilayered Networks. Neural Networks, 4:15-25, 1991.

[3] S. Amari. Dynamic of pattern formation in lateral-inhibition type neural fields. Biological Cybernetics, 27:77-78, 1977.

[4] D. H. Ballard. Cortical connections and parallel processing: Structure and function. Behavioral and Brain Sciences, 9:67-120, 1986.

[5] D. H. Ballard, M. M. Hayhoe, P. K. Pook, and R. P. N. Rao. Deictic codes for the embodiment of cognition. Behavioral and Brain Sciences, 20:723-767, 1997.

[6] V. Bringuier, F. Chavane, L. Glaeser, and Y. Fregnac. Horizontal propagation of visual activity in the synaptic integration field of area 17 neurons. Science, 283:695-699, 1999.

[7] R. Brooks. Intelligence without representatio. Artificial Intelligence, 47:139-159, 1991.

[8] Y. Burnod. An adaptive neural network : the cerebral cortex. Masson, 1989.

[9] K. Doya. What are the computations of the cerebellum, the basal ganglia and the cerebral cortex? Neural Networks, 12:961-974, 1999.

[10] J. Fix, N. Rougier, and F. Alexandre. From physiological principles to computational models of the cortex. Journal of Physiology, 101(1-3):3239, May 2007.

[11] J. Fix, N.P. Rougier, and F. Alexandre. A top-down attentional system scanning multiple targets with saccades. In From Computational Cognitive Neuroscience to Computer Vision, CCNCV 2007, 2007. 
[12] J. Fix, J. Vitay, and N. P. Rougier. A computational model of spatial memory anticipation during visual search. In Proceedings of the Third Workshop on Anticipatory Behavior in Adaptive Learning System (ABIALS 2006), 2006.

[13] J. M.D Fuster. The Prefrontal Cortex : Anatomy, Physiology, and Neuropsychology of the Frontal Lobe. Lippincott Williams and Wilkins Publishers, 1997.

[14] J. J. Gibson. The Ecological Approach to Visual Perception. Houghton Mifflin, Boston, 1979.

[15] M. A. Goodale and G. K. Humphrey. The objects of action and perception. Cognition, 67:181-207, 1998.

[16] S. Grossberg and G. Swaminathan. A laminar cortical model for 3d perception of slanted and curved surfaces and of $2 \mathrm{~d}$ images: development, attention and bistability. Vision Research, 44:1147-1187, 2004.

[17] D. Jancke, F. Chavane, S. Naaman, and A. Grinvald. Imaging cortical correlates of illusion in early visual cortex. Nature, 428:423-6, 2004.

[18] E. Koechlin, J.-L. Anton, and Y. Burnod. Bayesian inference in populations of cortical neurons: a model of motion integration and segmentation in area mt. Biological Cybernetics, 80:25-44, 1999.

[19] T. Kohonen. Self-Organization and Associative Memory, volume 8 of Springer Series in Information Sciences. Springer-Verlag, 1989.

[20] E. Korner, M.-O. Gawaltig, U. Korner, A. Richter, and T. Rodemann. A model of computation in neocortical architecture. Neural Networks, 12:989-1005, 1999.

[21] O. Ménard and H. Frezza-Buet. Multi-map self-organization for sensorimotor learning: a cortical approach. In IEEE International Joint Conference on Neural Networks, 2003.

[22] R. Miikkulainen, J. A.Bednar, T. Choe, and J. Sirosh. Self-organization, plasticity, and low-level visual phenomena in a laterally connected map model of the primary visual cortex. Psychology of Learning and Motivation, 1996. 
[23] V. Mountcastle. The Mindful Brain (Gerald M. Edelman and Vernon B. Mountcastle, eds.), chapter An Organizing Principle for Cerebral Function: The Unit Model and the Distributed System. Cambridge, MA: MIT Press., 1978.

[24] J. Norman. Two visual systems and two theories of perception. Behavioral and Brain Sciences, 25:73-144, 2002.

[25] J. K. O'Regan and A. Noe. A sensorimotor account of vision and visual consciousness. Behavioral and Brain Sciences, 24:939-973, 2001.

[26] M.I. Posner and Y. Cohen. Components of visual orienting. In H. Bouma and D. Bouwhuis, editors, Attention and performance, volume X, pages 531-556. Lawrence Erlbaum, 1984.

[27] G. Rizzolati, L. Riggio, I. Dascola, and C. Ulmita. Reorienting attention accross the horizontal and vertical meridians. Neuropsychologia, 25:3140, 1987.

[28] G. Rizzolatti. The mirror neuron system and its function in humans. Anat Embryol, 210(5-6):419-21, 2005.

[29] N.P. Rougier. Dynamic neural field with local inhibition. Biological Cybernetics, 94(3):169-179, March 2006.

[30] L. R. Squire. Memory and the hippocampus: a synthesis from findings with rats, monkeys, and humans. Psychological Review, 99:195-231, 1992.

[31] J. G. Taylor. Neural networks for consciousness. Neural Networks, 10(7):1207-1225, 1997.

[32] J. G. Taylor. Neural bubble dynamics in two dimensions. Biological Cybernetics, 80:5167-5174, 1999.

[33] A. Treisman and G. Gelade. A feature-integration theory of attention. Cognitive Psychology, 12:97-136, 1980.

[34] J. Vitay, N. Rougier, and F. Alexandre. A distributed model of spatial visual attention. In S. Wermter, G. Palm, and M. Elshaw, editors, Biomimetic Neural Learning for Intelligent Robots: Intelligent Systems, 
Cognitive Robotics, and Neuroscience, volume 3575 of Lecture Notes in Computer Science, pages 54-72. Springer, Aug 2005.

[35] J. C. Wiemer. The time-organized map algorithm: Extending the self-organizing map to spatiotemporal signals. Neural Computation, 15:1143-1171, 2003.

[36] H.R. Wilson and J.D. Cowan. Excitatory and inhibitory interactions in localized populations of model neurons. Biophys. J., 12:1-24, 1972. 


\begin{tabular}{|c|c|c|}
\hline Principle of information processing & Cortical layer & Information flow \\
\hline \hline Sensory filtering & 4 & Feedforward and Thalamic input \\
\hline Sequence processing & $2 / 3$ & Lateral Intra-Area \\
\hline Multimodal integration & $2 / 3$ & Cortico-cortical \\
\hline Output & $5 / 6$ & Feedback and extracortical output \\
\hline
\end{tabular}

Table 1: 
Caption for Table 1: Fundamental principles of information processing are applied to various information flows in the laminar organization of the cortex. 


\begin{tabular}{|c|c|c|}
\hline Task & Identification & Location \\
\hline \hline Associative cortex & Temporal & Parietal \\
\hline Linking sensory pole with & Limbic pole & Body pole \\
\hline Extracts hints related to & Emotional value & Position in space \\
\hline
\end{tabular}

Table 2: 
Caption for Table 2: Two apparently different tasks (identification and location) rely on similar principles. 


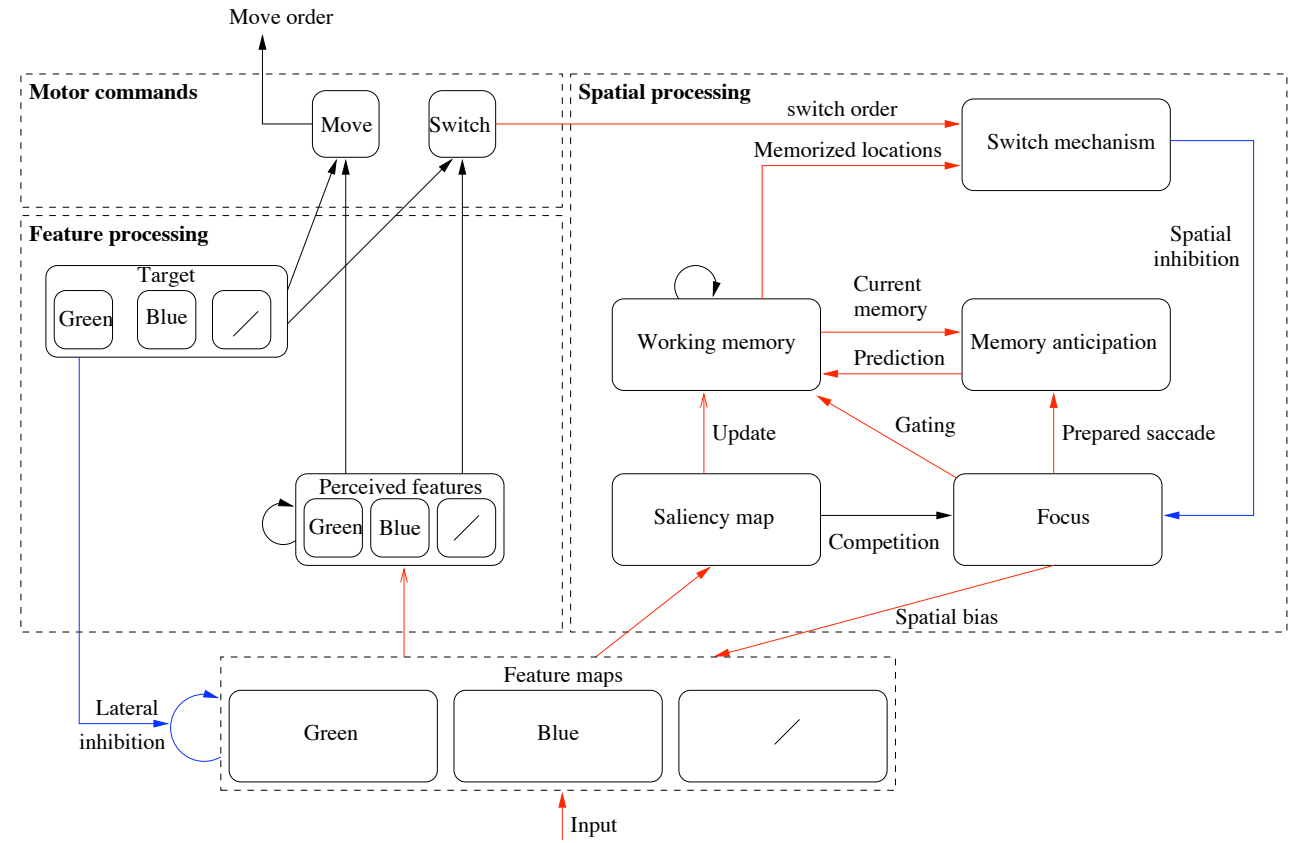

Figure 1: 
Caption for Figure 1: General structure of a model of visual overt and covert attention: feature and spatial processing are respectively performed in models of temporal and parietal cortical axes. Features are extracted in the Feature maps and combined in the Saliency map. The Focus map selects only one pattern because it is salient or because it is desired thanks to the monitoring of the Feature processing axis, encoding Target and Perceived features. The Working memory maps memorize the patterns that have been explored (inhibition of return) and the Memory anticipation map anticipates eye movements and update the Working memory. In the covert mode, the Switch mechanism inhibits the Focus map to put the focus on the next selected target. In the overt mode, a camera movement is performed if the target corresponds to the perceived feature. In accordance with a unified theory of cortical processing, all these apparently different processing are in fact produced by maps of cortically inspired units driven by identical functioning rules. 
move Splitch

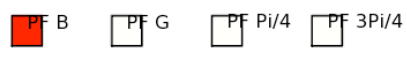

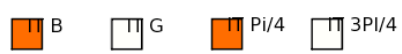
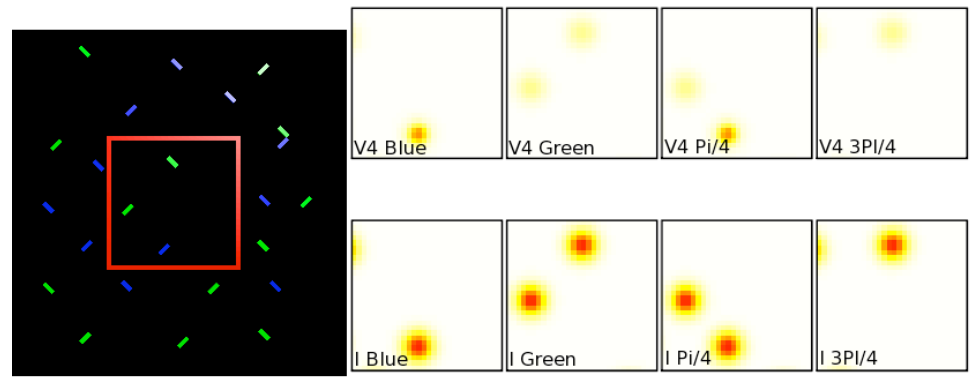

Figure 2:
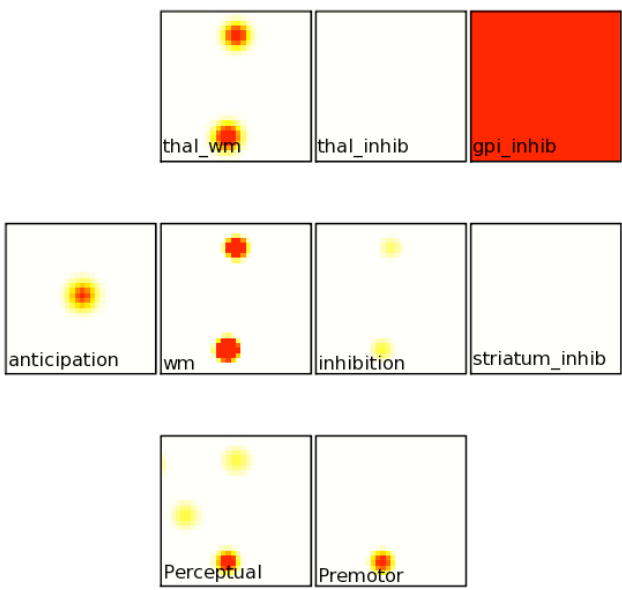

igure 2 . 
Caption for Figure 2: Screenshot of the model. This is an implementation with maps and units of the functional circuit presented in Figure 1. Left part: The perceived world is in the red square which can be moved (overt attention) or can be internally explored (switch: covert attention). Observed (bottom row) and desired (middle row) hints of color (Blue; Green) and orientation $(\mathrm{Pi} / 4 ; 3 \mathrm{Pi} / 4)$. Upper row: Move or Switch order. Right part: implementation in maps of cortically-inspired units of anticipation, working memory and inhibition of return for the appropriate analysis of the scene. The perceptual map stands for the Saliency map; the Premotor map stands for the Focus map. The Working memory is composed of wm and thalwm maps. The four upper left maps related to inhibition correspond to the Switch mechanism, not described here. Refer to the text and to $[12,11,10]$ for details. 